\title{
Modeling Oblique Rotators: Magnetospheres and Winds
}

\author{
Steven N. Shore
}

Department of Physics and Astronomy, Indiana University South Bend, 1700 Mishawaka Ave., South Bend, IN 46634-7111 USA

\begin{abstract}
The upper main sequence chemically peculiar (CP) stars display evidence of trapped circumstellar gas and nonspherical outflows. These stars are also known to possess strong magnetic fields that are often highly inclined to the rotational axis. Their phenomenology can be understood by using the oblique rotator model, which has successfully accounted for the observed behavior of the cooler CP stars. This paper reviews some features of the oblique rotator model, in which the magnetic field is assumed to provide a rigid framework for the structuring of the stellar and circumstellar gas. Corotation of circumstellar plasma is enforced out to the Alfven radius in the magnetic equatorial plane, while for the hotter stars, a radiatively driven wind emerges from the magnetic polar caps. Some observable consequences of the model are discussed, especially the $\mathrm{H} \alpha$ and ultraviolet resonance line absorption and emission periodic variability that has been observed in the He-peculiar stars and nonthermal radio emission. Magnetospheres may also be present in $\mathrm{O}$ stars, e.g. $\theta^{1}$ Ori $\mathrm{C}$, and in the Herbig Ae/Be stars.
\end{abstract}

\section{Introduction}

The phenomenological oblique rotator model is actually as old as mathematical astronomy itself, having been introduced by Eudoxus (ca. -350) to explain the planetary motions (see Neugebauer 1983). For more recent applications, however, the history dates back to the explanation of variable stellar spectra and magnetic fields observed in the upper main sequence chemically peculiar (CP) stars (Deutsch 1958, 1970). In its most basic form, the model contains only two adjustable geometric parameters that permit transformation between the magnetic and observer's frames. The magnetic fields in the CP stars are frozen into the stellar envelope and not actively generated by contemporaneous dynamos. The field thus provides a rigidly rotating ${ }^{1}$ coordinate system. The first parameter is $i$, the inclination of the rotational axis to the line of sight. This is trivially derived, since model atmosphere studies and parallax measurements combine to provide the stellar radius and

1 The stability of the surface features is attested to by the longterm regularity of the photometric variations of a few Ap stars, especially $\alpha^{2}$ CVn, which has maintained the same period since it was first observed photometrically early in this century (Pyper 1966). 
thus $v_{e q}=50.6\left(R_{s} \operatorname{tar} / R_{\odot}\right)(P / \text { days })^{-1} \mathrm{~km} \mathrm{~s}^{-1}$, where $P$ is the rotation period in days and $R_{\star}$ is the stellar radius. ${ }^{2}$ The other model parameter is $\beta=\cos ^{-1} \hat{\omega} \cdot \hat{\mathbf{B}}$, the obliquity of the magnetic field symmetry axis, $\hat{\mathbf{B}}$, to the rotational axis, $\hat{\omega}$. For CP stars, although the mechanism is not thoroughly understood (Moss 1990$)^{3}$, the field is inclined to the rotation axis. We can therefore write the simple coordinate transformation of rotation matrices, $\mathcal{R}$, as $\mathbf{x}=\mathcal{R}(\omega) \mathcal{R}(i) \mathcal{R}(\beta) \mathbf{x}^{\prime}$. Here $\mathbf{x}^{\prime}$ is the magnetic coordinate system and $\mathbf{x}$ is the observer's frame, which is centered on the visible hemisphere and over which all averages are taken including the limb darkening, $\Lambda(\theta)$. For an unresolved surface, the model treats all quantities distributed over the photosphere by averaging over the surface brightness, given by the series expansion:

$$
\xi\left(\theta^{\prime}, \phi^{\prime}\right)=\sum_{l m} a_{l m} Y_{l m}\left(\theta^{\prime} \phi^{\prime}\right)
$$

where $\theta$ and $\phi$ are the meridional and azimuthal angles, respectively, and $Y_{l m}$ is a spherical harmonic of order $(l, m)$. Thus, $Q(\theta, \phi)$ transforms into a mean quantity that is a function only of phase:

$$
<Q(\Phi)>=\int d \Omega Q(\theta, \phi) \Lambda(\theta) \xi(\theta, \phi)
$$

where $\Omega$ is the subtended solid angle, $A$ and $\xi$ are normalized in the observer's frame, and $\Phi$ is the rotational phase. This is essentially the basis for Doppler imaging since, for a rigid rotator, each point on the surface maps to a position in the line profile whether due to velocity alone or to the Zeeman effect and velocity acting together (Deutsch 1970; Mihalas 1973; Mesessier et al. 1979; Rice et al. 1989). Doppler imaging using maximum entropy reconstructions of stellar surfaces have been successful in providing statistically robust, modelfree snapshots of elemental and flux distributions (cf. Hatzes et al. 1989; Piskunov and Rice 1993 and refs. therein), although the resultant map is hard to quantitatively associate with any feature of the magnetic field geometry or polarity (see however Semel 1989).

The line of sight (longitudinal) magnetic field, $B_{\text {eff }}$, varies due to the rotation and the obliquity is provided by measurements of $r=B_{\text {eff, }+} / B_{\text {eff,- }}$, the ratio of the projected magnetic field extrema (e.g. Borra, Landstreet, and Mestel 1982). The combination of field distribution and velocity shifting of the Zeeman components produces the cross-over effect that yields important model information (e.g. Mathys 1995). Finally, restricting the distribution to a dipole in the magnetic frame, an axisymmetric distribution produces a double minimum in the light curve that corresponds to magnetic equatorial

${ }^{2}$ Additional support for this picture comes from measurements of the phasing and variations of pulsation of the rapidly oscillating Ap stars (roAp) (e.g. Kurtz 1990).

${ }^{3}$ This obliquity is also observed for virtually all planets in the Solar System, although the explanation is equally elusive there. 
crossing, so that $\cos (\Delta \Phi / 2)=\cot i \cot \beta$, where $\Delta \Phi$ is the phase separation of the mimima. This simplification in the geometry is especially important for diagnosing and modeling magnetospheres.

\section{Magnetospheres}

There are several signatures of magnetospheres in the CP stars. In the Hestrong stars, Balmer line emission is modulated with the rotational phase (e.g. Nakajima 1985; Bolton 1994). The periodic $\mathrm{H} \alpha$ variations in the O7 V star $\theta^{1}$ Ori C (Stahl et al. 1996) seem to be similar. The best studied case, HD 37479 $=\sigma$ Ori $\mathrm{E}$, which is not axisymmetric in either the magnetic or rotational frame (e.g. Hunger et al. 1993; Bolton 1994; Groote and Hunger 1997), also shows periodic shell-like absorption on the highest Balmer series lines when $\mathrm{H} \alpha$ is strongest. Some He-weak stars also display variable $\mathrm{H} \alpha$ emission that is modulated on the rotational phase (see Shore et al. 1998 and refs. therein) (especially HD $79158=36$ Lyn and HD 35502). It therefore seems that many of the hotter members of the He-weak sequence of chemically peculiar stars possess magnetospheres.

The unambiguous evidence of magnetospheric plasma is the detection of enhanced, variable C IV and Si IV resonance line absorption in the CP stars (Shore 1987; Shore, Brown and Sonneborn 1987; Shore et al. 1990; Shore 1990). This behavior has also been detected in the O7 V star $\theta^{1}$ Ori C (Walborn and Nichols 1994). The phase relation for the variable CP star line profiles clearly shows that the strongest absorption occurs when the magnetic equator crosses the line of sight. The profiles are only moderately variable in symmetry, indicating that the gas is constrained to corotate to large distance - the extent can be determined by the occultation of the magnetospheric gas by the stellar disk (Fig. 1). The geometry is usually dipolar, but here one star deserves special mention: HD 37776. This is the only known He-strong star with a predominantly quadrupolar surface field (Thompson and Landstreet 1985). The C IV variations, although precisely periodic over the magnetic cycle, do not correlate well with the projected line of sight field strength.

A clue to the geometry of the circumstellar plasma comes from the slowly rotating He-strong stars: the stars that show emission (HD 5 and HD 96446) have constant strong magnetic fields while those showing strong, constant $\mathrm{C}$ IV absorption, HD 60344 and HD 133518, show no detectable fields ${ }^{4}$ (Shore and Brown 1990).

In addition, a number of CP stars are nonthermal radio emitters (Linsky, Drake, and Bastien 1992; Leone, Umana, and Trigilio 1996). Phillips and Lestrade (1988) have even used VLBI to place limits of about $6 R_{\star}$ on the

${ }^{4}$ At this meeting, Mathys mentioned observations of HD 96446 that seem to present a problem for the simple unified picture I have just described. This star shows periodic variations of the magnetic field that are not consistent with the geometry implied by the emission line profile and hint at a non-dipolar geometry. 
size of the emission regions in HD 37017 and HD 37479, two prototypical He-strong stars. For the He-weak and Si stars, Linsky et al. (1992) obtained many detections, while Drake, Linsky, and Bookbinder (1994) find for a sample of 23 candidates that no non-magnetic CP stars are radio sources and therefore argue that such stars lack magnetospheres. Linsky et al., concentrating exclusively on radio measurements, find a general scaling for the 6 cm luminosity, $L_{6 \mathrm{~cm}} \sim \dot{M}^{2 / 5} B \omega^{1 / 3}$, where $B$ is the surface field, $\dot{M}$ is the mass loss rate, and $\omega$ is the rotational frequency. There is considerable uncertainty in the mass loss rates for the He-weak stars compared with the He-strong stars so their scaling relation is only preliminary. One advantage of the UV resonance line measurements is that it provides direct information about the density and velocity of the material in the circumstellar plasma. The implicated mechanism for emission is gyrosynchrotron radiation (Linsky 1993). Havnes and Goertz (1984) suggested that the emission is powered by reconnection at the Alfven surface. The formation of a compact disk, of the sort discussed by J. Bjorkman and Cassinelli at this meeting, results from compression in the magnetic equatorial plane, with outflow and heating occurring due to the resulting pressure gradient from the equatorial collisions.

For cooler B stars, a centrifugally driven wind is also a possible source for circumstellar plasma (Mestel 1968) since the He-weak stars do not possess strong radiatively driven winds with which to supply the circumstellar gas. In this case, the rapidly rotating magnetic stars might then be expected to show more circumstellar gas, and there should be a strong dependence of the density of the magnetospheric gas on the rotation frequency and magnetic field strength (Mestel 1968; Nerney and Suess 1987; Shore and Brown 1990). It is therefore something of a surprise that we do not see any evidence for so simple a picture among the helium-weak and silicon stars. For the He-strong stars, the periods are pretty uniform, from about 1 to 10 days. The He-weak stars show a larger range of rotational periods with which the magnetospheres are not well corelated; e.g. HD $5737=\alpha$ Scl has a 21 day period and displays a similar variability to HD 142301 , which has a period of $\approx 1.5$ days. In addition, the magnetospheric C IV strength seems not to depend on magnetic field strength or oblique rotator parameters.

Theoretically, the magnetosphere is a closed surface whose extent is the Alfven radius (e.g. Dressler 1983; Michel 1991). This is related to the intrinsic properties of the star through $R_{A} \sim B^{1 / 3}\left(f v_{\infty}\right)^{1 / 6} \dot{M}^{-1 / 6} \omega^{-1 / 3} R_{\star}$, where $f v_{\infty}$ is the fraction of the terminal velocity of the wind (cf. Shore 1987, 1993). As observed for the outer planets, such as Jupiter (Dressler 1983), the circmstellar plasma is forced into approximate corotation out to $R_{A}$, and inertial effects concentrate matter into a disk in the magnetic equatorial plane. This should lead to formation of a current sheet. In light of the outflow that is expected in the hottest stars, this sheet could evolve by driven reconnection. Because its symmetry plane does not coincide with the rotational equator, 
the plasma is forced into a periodic oscillation on the rotational timescale. For planetary magnetospheres, which are strongly interacting with the solar wind, this produces reconnection in the magnetotail with attendant acceleration of electrons (cf. Nishida 1984). It is thus reasonable to expect reconnection to occur in CP star magnetospheres, leading to sporadic variations of the radio emission and local heating due to turbulence. In addition, the backward flowing particles will dissipate their energy in the stellar atmosphere, producing an auroral ring - a region in a planetary atmosphere that is strongly heated by particle precipitation from the current sheet - and providing the hot ions that are responsible for C IV absorption in the cooler He-weak stars.

A fundamental difference between stellar and either Solar System magnetospheres or those in cataclysmic variables is the absence of external flows in the isolated star case. The CP stars are self-structured, in the sense that the heating and shear is due to internal processes, such as stellar winds and reconnection. To date, the most comprehensive models for heating and dynamics of the trapped circumstellar plasma in magnetic CP stars has been by Babel and Montmerle (1997a, 1997b), following formalism by Havnes and Goertz (1984), who use an analogy to the terrestrial magnetosphere. Babel and Montmerle successfully explain the X-ray emission from HD 34452 as arising from shocks within the magnetosphere. A problem with this picture may be that this star does not show enhanced C IV absorption, while other stars that do show this are not X-ray emitters.

As an example of the generic behavior expected for the $\mathrm{H} \alpha$ emission, I show in Fig. 1 the variation of the line profile with phase for a simple model with a disk-like distribution for the emission $j\left(\theta^{\prime}\right) \sim P_{2}\left(\theta^{\prime}\right)^{n}$ where $P_{2}$ is the Legendre polynomial in the magnetic frame and $n$ is a free parameter describing the flattening of the magnetosphere (Shore and Brown 1990). Figure 2 shows the integrated emission strength as a function of phase. The parameters were chosen to reproduce the variations of $\theta^{1}$ Ori C (Stahl et al. 1996). The width of the line provides the Alfven radius and requires at least a kilogauss field to maintain corotation to around $4 R_{\star}$. Such a field has not, however, been detected, although Babel and Montmerle (this meeting) argue that a much weaker field can yield the observed X-rays. The integrated flux curve is generic (Fig. 2) and any oblique magnetospheric distribution will produce the same behavior. The C IV lines, however, indicate a very thick region and again require a large field to maintain the structure. Yes, this is a puzzle.

\section{Non-spherical Mass Loss}

In the hottest stars, radiation pressure is expected to drive outflows. That is what much of this meeting has been about. The imposition of a strong magnetic field, however, necessarily renders the flow aspherical. The magnetosphere is a "dead zone" for outflow, so any mass loss can only occur from 

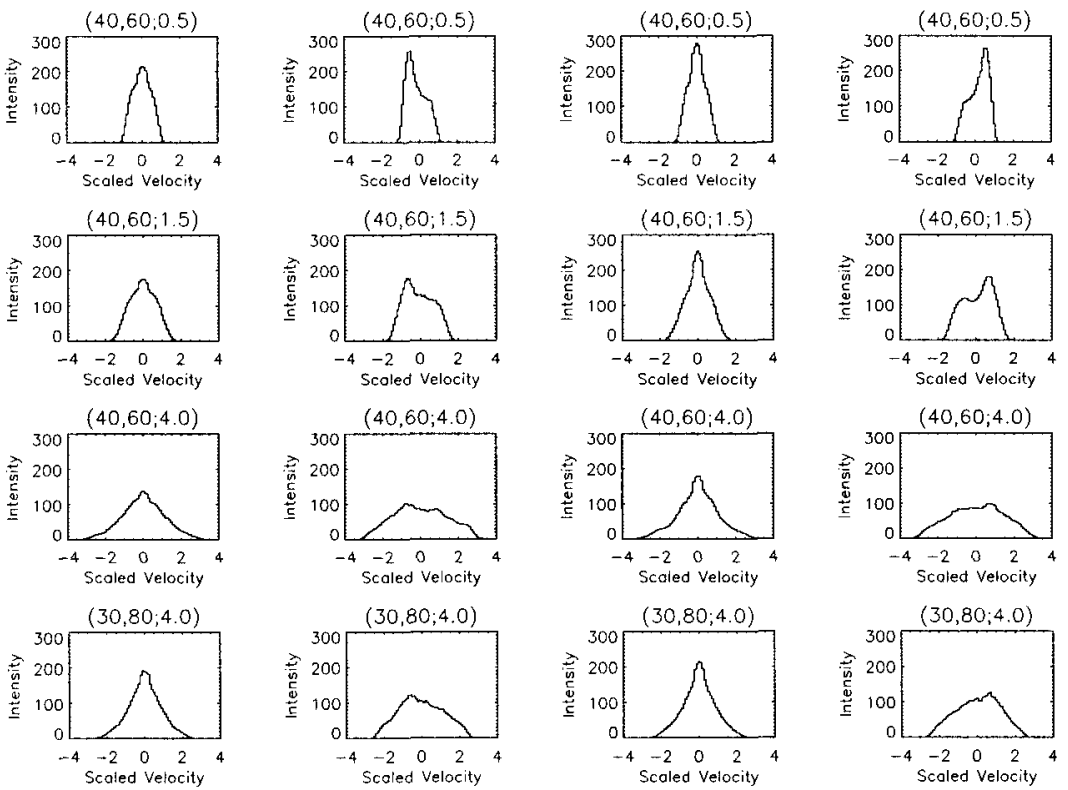

Fig. 1. Sample line profile variations, labeled by $(i, \beta)$ and the outer radius $\left(\mathrm{R}_{A}-\mathrm{R}_{\star}\right)$. Notice the relative insensitivity to oblique rotator parameters although strong dependence on radius. The profiles show phases $0.0,0.25,0.5$, and 0.75 , respectively.

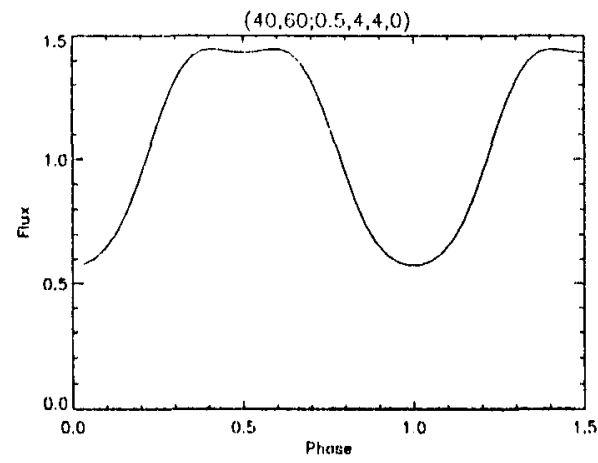

Fig. 2. Integrated line flux curve for $i=40, \beta=60$ (see Fig. 1). Magnetic polar traverse is at $\Phi=0^{p} .5$. 
latitudes whose field lines close outside the Alfven surface. This restricts the opening cone for the flow to $\Delta \theta=\sin ^{-1}\left(R_{\star} / R_{A}\right)^{1 / 2}$. Heating may be provided by plasma turbulence or by shocks. Nonthermal radio emission is observed in several stars, notably the strongest field He-strong stars (especially HD $37479=\sigma$ Ori E), but it also extends to the cooler He-weak stars with the strongest fields. While the He-strong stars appear to show rotational emission modulation, this is not as clear for the He-weak emitters. ${ }^{5}$

The strongest case for a collimated outflow is HD 21699 (Brown et al. 1985). This He-weak $\mathrm{Si}$ star is unique in showing the strongest $\mathrm{C}$ IV absorption when the strongest magnetic pole is in the line of sight. In addition, the absorption is strongly asymmetric, which is not seen in any of the variable magnetospheric stars, and the terminal velocity is consistent with corotation out to $R_{A}$. This star is a member of the $\alpha$ Per cluster so its age is known and the star is clearly on or near the ZAMS, but not pre-main sequence. ${ }^{6}$ Among the He-strong stars, HD 58260 and HD 96446 show only nonvariable, asymmetric emission. There is an extended red wing but the blueward side of the line is truncated at the rest wavelength. Shore and Brown (1990) schematically model this as a combination of a magnetosphere and polar outflow (see their fig. 10).

For collimated flows, the solid angle of the polar cone is relatively small so the normal P Cygni emission line wings are absent. The simplest models (Kunacz 1984; Brown et al. 1985; Shore et al. 1994) produce only absorption troughs whose radial velocity and depth change with rotational phase. The absorption disappears for transverse presentation of the flow to the line of sight. Thus, for orthogonal rotators such as the He-strong stars HD 60344 and HD 133518, there is no contribution from the wind to the line profile. The weakness of the emission wing may also explain the pbserved profiles in some of the Herbig $\mathrm{Ae} / \mathrm{Be}$, such as $\mathrm{AB}$ Aur.

Acknowledgments: I wish to thank the SOC for their kind invitation to this wonderful meeting and Jacques Babel ${ }^{7}$, Joe Cassinelli, Henny Lamers, Gauthier Mathys, Stan Owocki, and Steve White for discussions during the sessions. Some of the work reported in this review is part of a larger study of the helium peculiar stars with David Bohlender, Tom Bolton, and Pierre North. IUE observations of the helium peculiar stars were supported by NASA through NAG5-2612 to IUSB.

${ }^{5}$ Linsky et al. find variable radio emission but the data set to date is too sparse to permit accurate phasing of the detections.

${ }^{6}$ Many stars in this intermediate spectral range that show anomalous C IV absorption turn out to be Herbig Ae/Be stars (cf. Imhoff 1994). These may show the combined effects of outflow and magnetospheres, but they have yet to be modeled within this picture.

7 ... even though we disagree on the nature of $\theta^{1}$ Ori C. 


\section{References}

Babel, J., Montmerle, T. 1997, A\&A, 323, 121

Babel, J., Montmerle, T. 1997, ApJ, 485, L29

Barker, P. L., Brown, D. N., Bolton, C. T., Landstreet, J. D. 1982, in Advances in Ultraviolet Astronomy: Four Years of IUE Research, ed. Y. Kondo, J. Mead, R. D. Chapman (NASA CP-2238), p. 589

Bolton, C.T. 1994, ApSS, 221, 95

Brown, D.N., Shore, S.N., Sonneborn, G. 1985, AJ, 90, 1354

Deutsch, A. 1958, in IAU Symp. 6 ed. A. Lehnert (North-Holland: Amsterdam) p. 209

Deutsch, A. 1970, ApJ, 159, 985

Drake, S.A., Abbott, D.A., Bastien, T.S., Bieging, J.H., Churchwell, E., Dulk, G., Linsky, J. L. 1987, ApJ, 322, 902

Drake, S.A., Linsky, J.L., Schmitt, J. H. M. M., Russo, C. 1994, ApJ, 420, 387

Drake, S. A., Linsky, J. L., Bookbinder, J. A. 1994, AJ, 108, 2203

Dressler, A. ed. 1983, The Jovian Magnetosphere (Cambridge Univ. Press: Cambridge)

Groote, D. Hunger, K. 1982, A\&A, 116, 64

Groote, D., Hunger, K. 1997, A\&A, 319, 250

Hatzes, A., Penrod, G. D., Vogt, S. S. 1989, ApJ, 341, 456

Havnes, O., Goertz, C.K. 1984, A\&A, 138, 421

Hunger, K., Groote, D. 1993, Peculiar versus Normal Phenomena in A-type and Related Stars, ed. F. Castelli, M. M. Dworetsky (ASP Conf. Series: San Francisco), p. 394

Imhoff, C. 1994, in First International Meeting on Herbig Ae/Be Stars eds. M. Perez, E. van den Heuvel (ASP Conf. Series: San Francisco)

Kurtz, D. W. 1990, ARAA, 28, 607

Leone, F., Umana, G., Trigilio, C. 1996, A\&A, 310, 271

Linsky, J.L. 1993, in Peculiar versus Normal Phenomena in A-type and Related Stars, ed. F. Castelli, M. M. Dworetsky (ASP Conf. Series: San Francisco), p. 507

Linsky, J.L., Drake, S.A., Bastien, T.S. 1992, ApJ, 393, 341

Mathys, G. 1995, A\&A, 293, 733

Megessier, C., Khokhlova, V. L., Ryabchikova, T. A. 1979, A\&A, 71, 295

Mestel, L. 1968, MNRAS, 138, 359

Michel, F. C. 1991, Theory of Neutron Star Magntospheres (Univ. of Chicago Press: Chicago)

Mihalas, D. 1973, ApJ, 184, 851

Moss, D. 1990, MNRAS, 244, 272

Nakajima, R. 1985, ApSS, 116, 285

Nerney, S., Suess, S. 1987, ApJ, 321, 355

Neugebauer, O. 1983, Astronomy and History: Selected Essays (Springer-Verlag: Berlin)

Nishida, A. 1984, in Magnetic Reconnection in Space and Laboratory Plasmas (AGU Geophys. Mono. 30) (American Geophysical Union: Washington DC) p.159

Phillips, R. B., Lestrade, J-F, 1988, Nature, 334, 329

Piskunov, N. E., Rice, J. B. 1993, PASP, 105, 1415 
Rice, J. B., Wehlau, W. H., Khokhlova, V. L. 1989, A\&A, 208, 179

Semel, M. 1989, A\&A, 225, 456

Shore, S.N. 1987, AJ, 94, 731

Shore, S.N. 1993, in Peculiar versus Normal Phenomena in A-type and Related Stars, ed. F. Castelli, M. M. Dworetsky (ASP Conf. Series: San Francisco), p. 528

Shore, S. N., Bolton, C. T., Bohlender, D. A., North, P. 1998, preprint

Shore, S.N., Brown, D.N. 1990, ApJ, 365, 665

Shore, S.N., Brown, D.N., Sonneborn, G. 1987, AJ, 94, 737

Shore, S.N., Brown, D.N., Sonneborn, G., Landstreet, J.D., Bohlender, D.A. 1990, ApJ, 348, 242

Stahl, O. et al. 1996, A\&A, 312, 539

Thompson, I. B., Landstreet, J. D. 1985, ApJ, 289, L9

Walborn, N. R., Nichols, J. S. 1994, ApJL, 425, L29

\section{Discussion}

H. Henrichs: Could it be that your $\theta^{1}$ Ori $\mathrm{C}$ magnetic field measurements were taken around a phase when the field was small?

G. Mathys: No magnetic field was detected in $\theta^{1}$ Ori $\mathrm{C}$ in about a dozen observations distributed in phase: this rules out the possibility of null measurements by chance.

G. Mathys: The observations of the He-strong star HD 96446 could probably be easily modelled, as you claim, if the star were an oblique rotator. However, as mentioned in my talk, the magnetic observations would in this case imply that HD 96446 should have an unrealistically small radius of less than $2 \mathrm{R}_{\odot}$. Therefore, the oblique rotator model must be questioned for this star.

S. Shore: This could be evidence of trapped polar oscillations, analogous to the roAp stars. Since HD 96446 should have $i$ and $\beta \sim 0$ (aligned, low inclination), it could be showing this (if the star can have an overstable mode or modes). Then the same should be seen for the emission line He-strong stars; but the He-strong stars with only C IV steady absorption should not show it.

S. White: I would like to comment that we see the magnetosphere in radio observations as well: a small number of stars have been observed over several rotational periods and they show a clear signal in the circular polarisation of the underlying rotating magnetic field (work by Jeremy Lim et al., in preparation).

S. Shore: Both the He-weak and He-strong stars show this sort of emission. What we have found in the Sco OB 1 sample is that the strong radio emitters are also strong magnetosphere stars. There are lots of ways of accelerating particles in these dynamical structures, especially $\mathrm{K}-\mathrm{H}$ instabilities in the boundary between the trapped and outflowing plasma. But the radio emission could also be the clue to the heating required to produce the excess ionisation in the coolest stars. 